\title{
Abundances in the planetary nebula NGC 6210
}

\author{
S. R. Pottasch ${ }^{1}$, J. Bernard-Salas ${ }^{2}$, and T. L. Roellig ${ }^{3}$ \\ 1 Kapteyn Astronomical Institute, PO Box 800, 9700 AV Groningen, The Netherlands \\ e-mail: pottasch@astro.rug.nl \\ 2 Center for Radiophysics and Space Research, Cornell University, Ithaca, NY 14853, USA \\ 3 NASA Ames Research Center, MS 245-6, Moffett Field, CA 94035-1000, USA
}

Received 13 January 2009 / Accepted 17 March 2009

\begin{abstract}
The spectra of the planetary nebula NGC 6210 is reanalysed using spectral measurements made in the mid-infrared with the Spitzer Space Telescope and the Infrared Space Observatory. The aim is to determine the chemical composition of this object. We also make use of IUE and ground based spectra. Abundances determined from the mid-infrared lines, which are insensitive to electron temperature, are used as the basis for the determination of the composition, which is found to differ somewhat from earlier results. The abundances found, especially the low value of helium and oxygen, indicate that the central star was originally of rather low mass, probably $\leq 1 M_{\odot}$. Abundances of phosphorus, iron, silicon, sodium, potassium and chlorine have been determined, some for the first time in this nebula. The electron temperature in this nebula is constant. The temperature, radius and luminosity of the central star is also discussed. It is shown that the luminosity is consistent with that predicted for a star of $0.9 M_{\odot}$. But the predicted nebular age is inconsistent with the observed kinetic age.
\end{abstract}

Key words. ISM: abundances - planetary nebulae: individual: NGC 6210 - infrared: ISM - stars: evolution

\section{Introduction}

NGC 6210 (PN G043.1+37.7) is a rather bright planetary nebula located at an unusually high galactic latitude and has only a small extinction. The nebula has an unusual morphology. It is usually classified as ellipsoidal, although Hajian et al. (1995) refer to it as "very amorphous and irregular". An HST photograph of the nebula is shown in Fig. 1. This is the brightest part of the nebula. The central star is clearly seen in the photograph; while the brightest part of the nebula lies to the north of the central star. Various small scale structures are also present. Most of the emission is present within a region of about $12^{\prime \prime} \times 8^{\prime \prime}$. This region is surrounded by a halo-like structure with a diameter about twice as large and of much lower surface brightness, less than $1 \%$ of the brighter parts of the nebula. A long exposure photograph of the nebula is shown in Fig. 2 where it can be seen that there are four arm-like extensions in the halo. Phillips \& Cuesta (1996) have shown that these features have a somewhat different spectrum than that of the main nebula. The integrated spectrum which is studied in this paper is essentially from the main part of the nebula, both because the halo emission is relatively small and because the size of the diaphragm limits the measurement to the central region.

Because the nebula is found 38 degrees above the galactic plane it has only a small extinction. It is probably a nearby nebula since most PNe are located close to the galactic plane. An expansion distance has been measured by Hajian et al. (1995) to be $1.57 \mathrm{kpc}$. Although this is an uncertain distance it is not an unreasonable one since it agrees with the distance found from

\footnotetext{
^ Based on observations with the Spitzer Space Telescope, which is operated by the Jet Propulsion Laboratory, California Institute of Technology and with ISO, an ESA project with instruments funded by ESA member states and with participation of ISAS and NASA.
}

the rms density. It is also similar to various statistical distances listed in Acker et al. (1992).

A bright central star can be seen in Fig. 1. It has a magnitude $V=12.66$ and $B=12.44$ (Acker et al. 1992). Mendez (1991) who measured the spectrum of the star calls it a hydrogen rich central star of type $\mathrm{O}(\mathrm{H})$. The hydrogen Zanstra temperature is about $50000 \mathrm{~K}$, and is somewhat less than the ionized helium Zanstra temperature which is close to $61000 \mathrm{~K}$. A discussion of the stellar continuum between $1500 \AA$ and $3000 \AA$ by Pottasch et al. (1978) gave the rather low temperature of $38000 \mathrm{~K}$; this is too low to ionize helium. We discuss the stellar temperature toward the end of this paper.

The purpose of this paper is to study the elemental abundances in this nebula with the help of mid-infrared spectra, in the hope that the chemical abundances will shed some light on the evolution of this nebula-central star combination. The most important previous abundances studies of this nebula are those of Liu et al. (2004a) and of Kwitter \& Henry (1998). Both of these groups use optical nebular spectra (taken by themselves) and ultraviolet IUE spectra. Liu et al. (2004a) also use ISO infrared measurements. Kwitter \& Henry (1998) find the abundance of the five elements He, C, N, O and Ne. Liu et al. (2004a) increase this number to eight by including $\mathrm{S}, \mathrm{Ar}$ and $\mathrm{Cl}$. Here the number of elements is increased to 13 by including $\mathrm{P}, \mathrm{Fe}, \mathrm{K}, \mathrm{Na}$ and an uncertain abundance of Si.

We have measured the spectrum of NGC6210 in the mid-infrared with the IRS spectrograph of the Spitzer Space Telescope (Werner et al. 2004). These infrared measurements are combined with the ISO infrared spectrum. The use of the mid-infrared spectrum permits a more accurate determination of the abundances. The reasons for this have been discussed in earlier studies (e.g. see Pottasch \& Beintema 1999; 


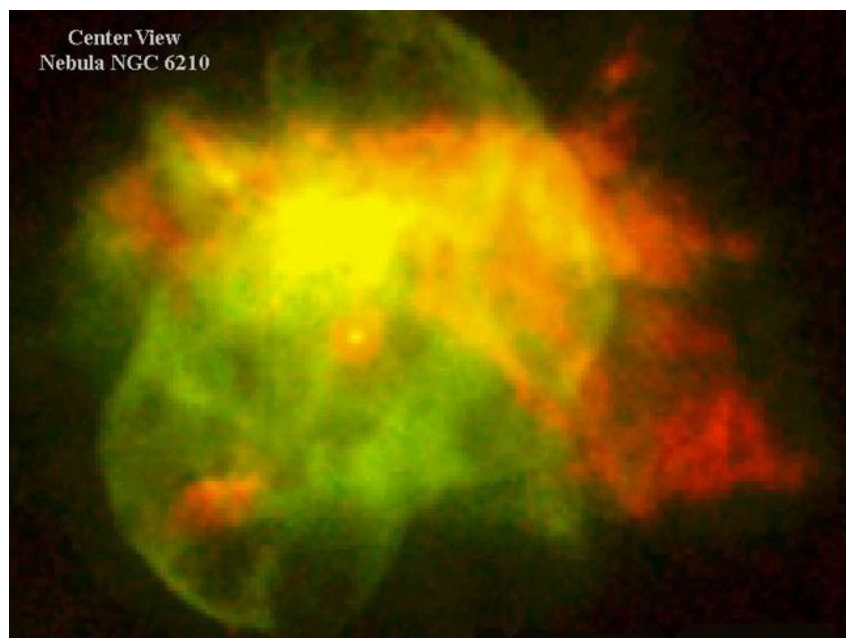

Fig. 1. An HST image of NGC 6210. The width of the photograph is $15.8^{\prime \prime}$. This is the brightest part of the nebula. Credit: NASA/courtesy of nasaimages.org

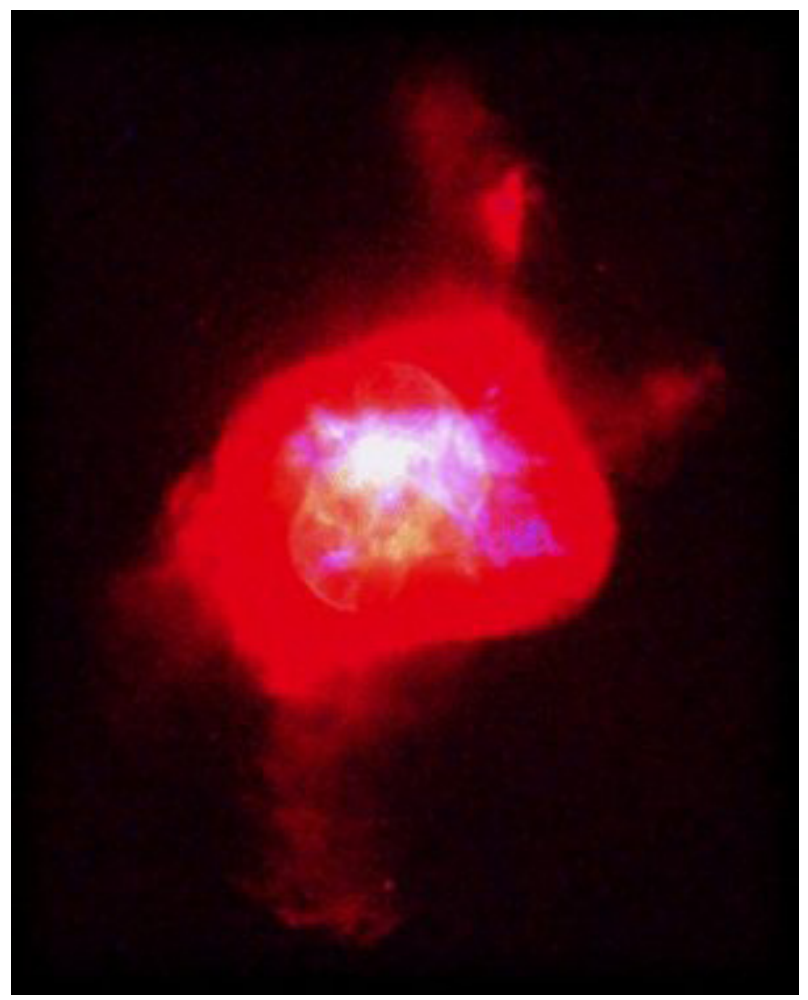

Fig. 2. A long exposure HST image of NGC 6210 emphasising the halo structure. The width of the photograph is $42^{\prime \prime}$. Credit: NASA/courtesy of nasaimages.org

Pottasch et al. 2000, 2001; Bernard Salas et al. 2001), and can be summarized as follows.

The most important advantage comes from the fact that the infrared lines originate from very low energy levels and thus give an abundance which is not sensitive to the temperature in the nebula, nor to possible temperature fluctuations. Furthermore, when a line originating from a high-lying energy level in the same ion is observed, it is possible to determine an average electron temperature at which the lines in that particular ion are formed. When this average temperature for many ions is determined, a plot of may be made of the electron temperature against ionization potential, which can be used to determine the average electron temperature of other ions as function of ionization potential.

Use of the infrared spectra have further advantages. One of them is that the number of observed ions used in the abundance analysis is approximately doubled, which removes the need for using large ionization correction factors (ICFs), thus substantially lowering the uncertainty in the abundance of some elements. A further advantage is that the extinction in the infrared is almost negligible, eliminating the need to include sometimes large correction factors. This is not very important in this nebula because the extinction is small. The number of elements that can be measured increases when including the infrared. In this nebula infrared lines of $\mathrm{Na}, \mathrm{P}, \mathrm{K}, \mathrm{Fe}$ and $\mathrm{Si}$ are measured.

The paper is structured as follows. First the Spitzer spectrum of NGC 6210 is presented and discussed (in Sect. 2), followed by a discussion of the ISO spectrum (Sect. 3). Then the intrinsic $\mathrm{H} \beta$ flux is determined using both the measurements of the infrared hydrogen lines and the radio continuum flux density (Sect. 4). The visible spectrum of the nebula is also presented in Sect. 4 together with a new reduction of the ultraviolet (IUE) spectrum. This is followed by a discussion of the nebular electron temperature and density and the chemical composition of NGC 6210 (Sect. 5). A comparison of the resulting abundances with those in the literature is given in Sect. 6 and the possible evolution is discussed in Sect. 7. This section also includes a discussion of nebular dust. In Sect. 8 the central star is discussed, followed by a general discussion and concluding remarks (Sect. 9).

\section{The IRS infrared spectrum of NGC 6210}

The observations of NGC 6210 were made using the Infrared Spectrograph (IRS, Houck et al. 2004) on board the Spitzer Space Telescope with AORkeys of 4109056 (on target) and 4109312 (background). The data were processed using the s15.3 version of the pipeline and using a script version of Smart (Higdon et al. 2004). The reduction started from the droop images which are equivalent to the $b c d$ images but lack stray-cross removal and flatfield. The tool irsclean was used to remove rogue pixels. The different cycles for a given module were combined to enhance the $\mathrm{S} / \mathrm{N}$. At this point the background images were subtracted to remove the sky contribution. Then the resulting HR images were extracted using full aperture measurements.

The great advantage of the IRS high resolution spectra compared to the ISO SWS spectra is the very high sensitivity of the IRS. Otherwise the two instruments are comparable. The IRS high resolution spectra have a spectral resolution of about 600 . The IRS high resolution instrument measures in two spectral ranges with two different modules: the short high module ( $\mathrm{SH}$ ) from $9.9 \mu \mathrm{m}$ to $19.6 \mu \mathrm{m}$ and the long high module (LH) from $18.7 \mu \mathrm{m}$ to $37.2 \mu \mathrm{m}$. The SH has a slit size of $4.7^{\prime \prime} \times 11.3^{\prime \prime}$, while the LH is $11.1^{\prime \prime} \times 22.3^{\prime \prime}$.

The IRS measurement of NGC 6210 was centered at $\operatorname{RA}(2000) 16^{\mathrm{h}} 44^{\mathrm{m}} 29.49^{\mathrm{s}}$ and $\operatorname{Dec}(2000)+23^{\circ} 47^{\prime} 59.7^{\prime \prime}$. This is almost the same as the value measured by Kerber et al. (2003) of RA(2000) $16^{\mathrm{h}} 44^{\mathrm{m}} 29.521^{\mathrm{s}}$ and $\operatorname{Dec}(2000)+23^{\circ} 47^{\prime} 59.55^{\prime \prime}$. Thus the IRS measurement was well centered on the nebula and both the LH and SH diaphragms measured the inner, brighter nebula. Since the LH diaphragm is larger, almost all of the inner nebula is seen in this diaphragm. Some of the nebula was missed in the $\mathrm{SH}$ measurement and therefore a correction must be made to bring the two measurements to the same scale. This can be done in several ways. First by making use of the fact that the two spectrographs had a small wavelength region in 
Table 1. IRS spectrum of NGC 6210.

\begin{tabular}{|c|c|c|c|}
\hline Identification & $\lambda(\mu \mathrm{m})$ & Intensity $^{\dagger}$ & $\mathrm{I} / \mathrm{H} \beta$ \\
\hline H I $(9-6)+[\mathrm{K} \mathrm{IV}]$ & 5.94 & $79.6 \pm 28$ & 0.72 \\
\hline Н І (12-7) & 6.77 & $17 \pm 0.4$ & \\
\hline [Ar II] & 6.98 & $83 \pm 4.5$ & 0.76 \\
\hline [Na III] & 7.31 & $53 \pm 2.9$ & 0.47 \\
\hline $\mathrm{HI}(6-5)$ & 7.47 & $301 \pm 17$ & \\
\hline [Ar III] & 8.99 & $1610 \pm 52$ & 14.7 \\
\hline [S IV] & 10.506 & $16700 \pm 250$ & 152 \\
\hline $\mathrm{HI}(9-7)$ & 11.305 & $48.2 \pm 1.2$ & \\
\hline [Cliv] & 11.757 & $90.1 \pm 3.7$ & 0.82 \\
\hline Н I $(7-6+11-8)$ & 12.368 & $114 \pm 4$ & \\
\hline$[\mathrm{Ne}$ II $]$ & 12.808 & $498 \pm 40$ & 4.55 \\
\hline H 1 (13-9) & 14.180 & $7.1 \pm 0.9$ & \\
\hline$[\mathrm{Cl} \mathrm{II}]$ & 14.359 & $4.74 \pm 0.6$ & 0.043 \\
\hline [Ne III] & 15.548 & $18500 \pm 1500$ & 169 \\
\hline Н I (10-8) & 16.210 & $12.4 \pm 2.4$ & \\
\hline [P III] & 17.885 & $22.7 \pm 2.2$ & 0.21 \\
\hline [S III] & 18.707 & $2820 \pm 120$ & 25.7 \\
\hline [Cl IV] & 20.310 & 48.7: \pm 6.6 & $0.45:$ \\
\hline [Ar III] & 21.814 & $79.5 \pm 10.5$ & 0.72 \\
\hline [Fe III] & 22.924 & $31.9 \pm 3.9$ & 0.29 \\
\hline [O IV] & 25.884 & $777 \pm 21$ & 7.10 \\
\hline$[\mathrm{Fe} \mathrm{III}]$ & 33.014 & $34.5: \pm 2.7$ & 0.31 \\
\hline [S III] & 33.470 & $890 \pm 10.7$ & 8.14 \\
\hline [Si II $]$ & 34.809 & $33.6 \pm 6.3$ & 0.30 \\
\hline [Ne III] & 36.001 & $1590 \pm 24$ & 14.5 \\
\hline
\end{tabular}

Intensities measured in units of $10^{-14} \mathrm{erg} \mathrm{cm}^{-2} \mathrm{~s}^{-1}$. The intensities below $19 \mu \mathrm{m}$ have been increased by a factor of 1.77 to bring them on the same scale as the LH intensities measured through a larger diaphragm. The intensities below $10 \mu \mathrm{m}$ are measured with the low resolution instrument and are brought to the same scale by matching the [S IV] and $[\mathrm{Ne}$ II] lines.

: Indicates an uncertain value.

The measured line intensity is given in Col. 3 . The last column gives the ratio of the line intensity to $\mathrm{H} \beta(=100)$.

common at about $19 \mu \mathrm{m}$. To make the continuum emission at this wavelength equal, the $\mathrm{SH}$ emission had to be increased by a factor 1.95 . But this factor may also be found by making use of the fact that both [ $\mathrm{Ne}$ III] and [Cl IV] have lines in both the $\mathrm{SH}$ and LH spectra and that the ratio of these lines has only a small density dependence. The ratio of the [Ne III] lines require a factor of 1.75 while the [Cl IV] lines require a still lower factor. But one of the [Cl IV] lines is weak and not much weight is given to these lines. A value of 1.77 is used to bring the $\mathrm{SH}$ intensities to the same scale as the LH values. There is a small uncertainty in this value, which has the consequence that uncertainty of the $\mathrm{SH}$ intensities, and hence the abundance of sulfur and sodium, is increased by $10 \%$.

Measurements have also been made with the low resolution spectrograph (called SL) (AORkey 4109056) which is in the form of a long slit with a width of $3.6^{\prime \prime}$. The spectrum is measured from $5.5 \mu \mathrm{m}$ to $14 \mu \mathrm{m}$ using a fix column extraction window of 4 ". The resolution is about a factor of 10 lower than the high resolution measurements. They are scaled so that the two strongest lines in the region of overlap, the [S IV] line at $10.506 \mu \mathrm{m}$ and the [Ne II] at $12.808 \mu \mathrm{m}$ have the same intensity. The measured emission line intensities are given in Table 1, after correcting the SH measurements by the factor 1.77 and the SL measurements by a factor of 2.40 . These values are given in the column labeled "intensity". The fluxes were measured using the Gaussian line-fitting routine and derived for each nod position independently. The uncertainty in the fluxes was assumed to be the larger of either the difference between the flux in the
Table 2. ISO spectrum of NGC 6210.

\begin{tabular}{lccc}
\hline \hline Identification & $\lambda(\mu \mathrm{m})$ & Intensity $^{\dagger}$ & $\mathrm{I} / \mathrm{H} \beta$ \\
\hline $\mathrm{H} \mathrm{I}(11-5)$ & 2.873 & 36.1 & \\
$\mathrm{H} \mathrm{I}(5-4)$ & 4.052 & 645 & \\
$\mathrm{H} \mathrm{I}(6-5)$ & 7.460 & 231 & \\
{$[\mathrm{Ar} \mathrm{III}]$} & 8.991 & 1120 & 12.7 \\
{$[\mathrm{~S} \mathrm{IV}]$} & 10.510 & 13450 & 152 \\
{$[\mathrm{Ne}$ II $]$} & 12.812 & 367 & 3.67 \\
{$[\mathrm{Ne}$ III $]$} & 15.554 & 17620 & 176 \\
{$[\mathrm{~S} \mathrm{III}]$} & 18.712 & 2300 & 23 \\
{$[\mathrm{Ar}$ III] } & 21.830 & 86 & 0.86 \\
{$[\mathrm{Fe} \mathrm{III}]$} & 22.928 & 45.3 & 0.45 \\
{$[\mathrm{O} \mathrm{IV}]$} & 25.890 & 569 & 5.7 \\
{$[\mathrm{~S} \mathrm{III}]$} & 33.478 & 1230 & 11.2 \\
{$[\mathrm{Ne}$ III] } & 36.010 & 1840 & 16.7 \\
{$[\mathrm{O} \mathrm{III}]$} & 51.800 & 16600 & 151 \\
{$[\mathrm{~N} \mathrm{III}]$} & 57.350 & 1650 & 15.0 \\
{$[\mathrm{O}$ I] } & 63.215 & 305 & 2.8 \\
{$[\mathrm{O} \mathrm{III}]$} & 88.353 & 5240 & 47.6 \\
\hline
\end{tabular}

$\dagger$ Intensities measured in units of $10^{-14} \mathrm{erg} \mathrm{cm}^{-2} \mathrm{~s}^{-1}$. No correction for extinction is necessary. The determination of $\mathrm{I} / \mathrm{H} \beta$ is discussed in the text.

The measured line intensity is given in Col. 3 . The last column gives the ratio of the line intensity to $\mathrm{H} \beta(=100)$.

nod positions, or the uncertainty in the fit. The last column gives the ratio of the intensity to $\mathrm{H} \beta$ where the $\mathrm{H} \beta$ is found from the strongest hydrogen line(s) measured in the IRS spectrum. It has been assumed that the theoretical ratio of the sum of the two hydrogen lines at $12.372 \mu \mathrm{m}$ to $\mathrm{H} \beta$ has a value of $1.04 \times 10^{-2}$, which is the value given by Hummer \& Storey (1987) for an electron temperature of $10000 \mathrm{~K}$. No correction for extinction is made since it is very small. This means that the value of $\mathrm{H} \beta$ through the LH diaphragm is $1.1 \times 10^{-10} \mathrm{erg} \mathrm{cm}^{-2} \mathrm{~s}^{-1}$ and the correction for reddening to $\mathrm{H} \beta$ is automatically included. Note that lines with wavelength less than $10 \mu \mathrm{m}$ are measured in the low resolution spectrograph.

\section{The ISO infrared spectrum of NGC 6210}

The ISO measurements of NGC 6210 are shown in Table 2. As can be seen from the table, the wavelength covered is considerably larger than the IRS measurements but because the sensitivity is lower only the stronger lines are seen. The intensities measured are given in Col. 3 of the table and are accurate to about $20 \%$. Two of the three SWS measurements, especially SWS02 TDT 28100801 which is essentially the data shown in the table, are centered at about the same position as the IRS measurements, namely RA(2000) $16^{\mathrm{h}} 44^{\mathrm{m}} 29.40^{\mathrm{s}}$ and $\operatorname{Dec}(2000)+23^{\circ} 48^{\prime} 00.4^{\prime \prime}$. But in spite of this, slightly less emission is being measured by the ISO. This is seen from the measurement of the hydrogen lines at 4.052 and 7.460 which indicate an $\mathrm{H} \beta$ flux of $0.88 \times 10^{-10} \mathrm{erg} \mathrm{cm}^{-2} \mathrm{~s}^{-1}$ as compared to the IRS measurement of $\mathrm{H} \beta=1.1 \times 10^{-10} \mathrm{erg} \mathrm{cm}^{-2} \mathrm{~s}^{-1}$. This is not a large difference and may simply be due to measurement error. But it could be related to the somewhat smaller ISO diaphragm below $12 \mu \mathrm{m}$. Thus for the ISO results a value of $0.88 \times 10^{-10} \mathrm{erg} \mathrm{cm}^{-2} \mathrm{~s}^{-1}$ is used below $12 \mu \mathrm{m}$, a value of $1.0 \times 10^{-10} \mathrm{erg} \mathrm{cm}^{-2} \mathrm{~s}^{-1}$ between this wavelength and $27 \mu \mathrm{m}$, and $1.1 \times 10^{-10}$ erg $\mathrm{cm}^{-2} \mathrm{~s}^{-1}$, the value deduced from the radio observations in the following section for the intensities made with the larger diaphragms above this wavelength. For the wavelengths above $50 \mu \mathrm{m}$ the LWS02 TDT28100802 measurement is used. 


\section{Extinction, visual and ultraviolet spectra}

There are several methods for obtaining the extinction. The dip at $\lambda 2200 \AA$ gives a value of $E_{B-V}=0.08(C=0.12)$ (Pottasch 1984). Using the $B$ and $V$ stellar magnitudes quoted above leads to a value $E_{B-V}=0.09$. The extinction obtained by comparing the observed and theoretical Balmer decrement gives a value $C=$ 0.075 (Kwitter \& Henry 1998) or $C=0.13$ (Liu et al. 2004a). Another accurate method is a comparison of radio emission with $\mathrm{H} \beta$ flux.

\subsection{The $6 \mathrm{~cm}$ radio emission}

The $6 \mathrm{~cm}$ flux density has been measured by Becker et al. (1991) and Gregory \& Condon (1991). Both of these authors find a value of $374 \mathrm{mJy}$. Using values of $T_{\mathrm{e}}$ and helium abundance determined below together with the equation quoted in Pottasch (1984) implies an $\mathrm{H} \beta$ flux of $1.10 \times 10^{-10} \mathrm{erg} \mathrm{cm}^{-2} \mathrm{~s}^{-1}$. Combining this with the measured value of $\mathrm{H} \beta=8.07 \times$ $10^{-11} \mathrm{erg} \mathrm{cm}^{-2} \mathrm{~s}^{-1}$ leads to a value of $C=0.14$, a value in close agreement with the values quoted above. This value $(C=0.14)$ will be used when necessary in this paper.

\subsection{The visual spectrum}

The visual spectrum has been measured by several authors. The highest resolution spectrum is by Liu et al. (2004a). These spectra are also the deepest. Reliable spectra have also been reported by Kwitter \& Henry (1998) and confirm the intensities of the stronger lines found by Liu et al. (2004a) which were taken by scanning over the entire nebula with a long slit. The spectrum of Liu et al. (2004a) refers to approximately the same region as the infrared measurements and will be used in this paper. The results are reproduced in Table 3 where Cols. 3 and 4 give the intensities measured and corrected for extinction relative to $\mathrm{H} \beta=100$ for the lines of interest in this paper. The error is estimated to be about $10 \%$ error for the strongest lines to about $30 \%$ for the weakest lines.

\subsection{The IUE ultraviolet spectrum}

There are 31 low resolution IUE observations of this nebula as well as seven high resolution observations. Most were taken with the large aperature $\left(10^{\prime \prime} \times 23^{\prime \prime}\right)$ with varying exposure times, but quite a few were taken with a small aperature ( $3^{\prime \prime}$ diameter). We find that the small aperature measurements are too noisy and we do not use them. The large aperture measurements cover most of the nebula when they are centered on the nebula. We have used some of the highest $\mathrm{S} / \mathrm{N}$ observations made with longer exposure times. Both high and low resolution measurements were used from both the short and longwavelength spectral regions. From the low resolution measurements we have used SWP01904, SWP54962 and LWR10117. The high resolution observations used were LWR14149, LWP02869 and SWP22384. We have checked that the strongest lines are not saturated by measuring these same lines on spectra with shorter exposure times. The results are shown in Table 4. The uncertainties are about the same as given by Kwitter \& Henry (1998), about $10 \%$ for the strongest lines and about $25 \%$ for the weaker lines. An exception to this is the O III line at $\lambda 3133$ which was only measured in low resolution because the high resolution spectra did not cover this region. The low resolution measurements show the C IV line at $\lambda 1548$ in emission, but the higher resolution measurements show that this
Table 3. Visual spectrum of NGC 6210.

\begin{tabular}{|c|c|c|c|}
\hline$\lambda$ & Ion & Meas.Intens. $\left.^{\dagger}\right)$ & Corr. Intens. \\
\hline 3726 & [O II] & 23.1 & 25.0 \\
\hline 3729 & [O II $]$ & 11.6 & 12.5 \\
\hline 3869 & [Ne III] & 80.9 & 86.7 \\
\hline 4101 & $\mathrm{H} \delta$ & 24.4 & 25.8 \\
\hline 4267 & $\mathrm{C}_{\mathrm{II}}$ & 0.327 & 0.341 \\
\hline 4340 & $\mathrm{H} \gamma$ & 45.7 & 47.5 \\
\hline 4363 & [O III] & 5.85 & 6.08 \\
\hline 4481 & $\mathrm{Mg}$ II & 0.19 & 0.020 \\
\hline 4686 & He II & 1.48 & 1.50 \\
\hline 4711 & [ArIV] & 1.16 & 1.173 \\
\hline 4740 & [Ar IV] & 1.52 & 1.534 \\
\hline 4861 & $\mathrm{H} \beta$ & 100 & 100 \\
\hline 5007 & [O III] & & 1084 \\
\hline 5276 & {$[\mathrm{Fe}$ VII $]$} & 0.015 & 0.015 \\
\hline 5517 & [Cl III] & 0.419 & 0.400 \\
\hline 5538 & {$[\mathrm{Cl}$ III] } & 0.492 & 0.469 \\
\hline 5755 & {$[\mathrm{~N} \mathrm{II}]$} & 0.417 & 0.393 \\
\hline 5876 & $\mathrm{He} \mathrm{I}$ & 14.6 & 13.7 \\
\hline 6312 & [S III $]$ & 1.19 & 1.093 \\
\hline 6563 & $\mathrm{H} \alpha$ & 304 & 276 \\
\hline 6584 & {$[\mathrm{~N}$ II $]$} & 20.6 & 18.7 \\
\hline 6717 & {$[\mathrm{~S}$ II $]$} & 2.42 & 2.185 \\
\hline 6731 & [S II $]$ & 4.01 & 3.618 \\
\hline 6794 & [K IV] & 0.029 & 0.026 \\
\hline 7135 & [Ar III] & 12.0 & 10.66 \\
\hline 7237 & [Ar IV] & 0.099 & 0.088 \\
\hline 7530 & [Cl IV] & 0.256 & 0.224 \\
\hline 9532 & [S III] & 44.3 & 49.6 \\
\hline
\end{tabular}

$\dagger$ References: all values are taken from Liu et al. (2004a) except for $\lambda 9532$ taken from Hippelein \& Munch (1981).

* This is a blend of $\lambda 3726$ and $\lambda 3729$ lines.

is a combination of absorption and emission; therefore we have not included this line.

The extinction correction was made by assuming a theoretical ratio for the He II line ratio $\lambda 1640 / \lambda 4686 \AA$ at $T=10000 \mathrm{~K}$ and an $N_{\mathrm{e}}$ of $10^{3} \mathrm{~cm}^{-3}$. The ratio of $\lambda 1640$ to $\mathrm{H} \beta$ can then be found using the $\lambda 4686 / \mathrm{H} \beta$ ratio in Table 3 together with the theoretical ratio, which leads to a value of the $\lambda 1640 \AA$ line as given in Col. (3) of Table 4. A further correction for extinction relative to $\lambda 1640 \AA$ is then made using the reddening curve of Fluks et al. (1994) but because of the small extinction this is never more than $20 \%$, which indicates the uncertainties of the UV intensities above the errors of measurement given above. The results are shown in the last two columns of Table 4.

\section{Chemical composition of the nebulae}

The method of analysis is the same as used in the papers cited in the introduction. First the electron density and temperature as a function of the ionization potential are determined. A constant electron density and temperature are found. Using these values the elemental abundances are derived.

\subsection{Electron density}

The ions used to determine $N_{\mathrm{e}}$ are listed in the first column of Table 5. The ionization potential required to reach this stage of ionization, and the wavelengths of the lines used, are given in Cols. 2 and 3 of the table. Note that the wavelength units are $\AA$ when 4 figures are given and microns when 3 figures are shown. The observed ratio of the lines is given in the fourth column; the 
Table 4. IUE Spectrum of NGC 6210.

\begin{tabular}{clcc}
\hline \hline$\lambda$ & Ion & \multicolumn{2}{c}{ Intensities } \\
\cline { 3 - 4 }$(\AA)$ & & $(1)$ & $\left(\mathrm{I} / \mathrm{H} \beta^{\dagger}\right)$ \\
\hline 1640 & He II & 40.6 & 9.75 \\
1661 & O III] & 6.08 & 1.45 \\
1665 & O III] & 15.0 & 3.60 \\
1750 & N III] & 11.3 & $2.7:$ \\
1882 & Si III] & 3.48 & 0.81 \\
1890 & Si III] & 3.90 & 0.90 \\
1906 & C III] & 61.0 & 14.7 \\
1909 & C III] & 43.5 & 10.5 \\
2297 & C III] & 9.0 & 2.3 \\
2325 & C II] & 6.1 & 1.54 \\
2471 & [O II] & 13.9 & 3.2 \\
2733 & He II & 1.2 & 0.24 \\
2764 & Fe II] & 2.4 & 0.48 \\
2830 & [Fe IV] & 4.2 & 0.83 \\
2836 & [Fe IV] & 4.2 & 0.83 \\
2946 & & 6.4 & 1.3 \\
3043 & & 1.8 & 0.35 \\
3048 & O III & 5.9 & 1.1 \\
3122 & & 3.6 & 0.70 \\
3133 & O III & $37:$ & $7.0:$ \\
\hline
\end{tabular}

(1) Measured intensity from low and high resolution spectra in units of $10^{-13} \mathrm{erg} \mathrm{cm}^{-2} \mathrm{~s}^{-1}$.

$\dagger$ Corrected for extinction and normalized to $\mathrm{H} \beta=100$.

Table 5. Electron density indicators in NGC 6210.

\begin{tabular}{lcccc}
\hline \hline Ion & $\begin{array}{c}\text { Ioniz. } \\
\text { Pot.(eV) }\end{array}$ & $\begin{array}{c}\text { Lines } \\
\text { used }\end{array}$ & $\begin{array}{c}\text { Observed } \\
\text { ratio }\end{array}$ & $\begin{array}{c}N_{\mathrm{e}} \\
\left(\mathrm{cm}^{-3}\right)\end{array}$ \\
\hline$[\mathrm{S} \mathrm{II}]$ & 10.4 & $6731 / 6716$ & 1.66 & 3600 \\
{$[\mathrm{O}$ II $]$} & 13.6 & $3626 / 3729$ & 1.99 & 3800 \\
{$[\mathrm{~S} \mathrm{III}]$} & 23.3 & $33.5 / 18.7$ & 0.487 & 2700 \\
{$[\mathrm{Cl}$ III $]$} & 23.8 & $5538 / 5518$ & 1.17 & 3400 \\
$\left.\mathrm{C}_{\text {III }}\right]$ & 24.4 & $1906 / 1909$ & 1.40 & $2000:$ \\
{$[\mathrm{A}$ IV $]$} & 40.7 & $4740 / 4711$ & 1.30 & 6800 \\
\hline
\end{tabular}

: Indicates uncertain value.

corresponding $N_{\mathrm{e}}$ is given in the fifth column. The temperature used is $10000 \mathrm{~K}$, but is unimportant since these line ratios are essentially determined by the density. No density is given from the Ne III lines because of the uncertainty of the $36 \mu \mathrm{m}$ line intensity. The density from the $\mathrm{C} \mathrm{III]}$ lines is uncertain because the measurements are uncertain.

There is no firm indication that the electron density varies with ionization potential in a systematic way. The electron density appears to be between 3000 and $4000 \mathrm{~cm}^{-3}$. The error is about $30 \%$. It is interesting to compare this value of the density with the rms density found from the $\mathrm{H} \beta$ line. This depends on the distance of the nebula for which a value of $1.6 \mathrm{kpc}$ is used, and on the angular size of the nebula. For this calculation a diameter of $16.5^{\prime \prime}$ is used. This gives an rms density of $3000 \mathrm{~cm}^{-3}$ for brighter regions. The agreement is very good although the error in the rms density is rather large. We will use a density of $3000 \mathrm{~cm}^{-3}$ in further discussion of the abundances, which will not be affected in an important way by the uncertainty in density.

\subsection{Electron temperature}

A number of ions have lines originating from energy levels far enough apart that their ratio is sensitive to the electron temperature. These are listed in Table 6 which is arranged similarly to
Table 6. Electron temperature indicators in NGC 6210.

\begin{tabular}{lcccc}
\hline \hline Ion & $\begin{array}{c}\text { Ioniz. } \\
\text { Pot.(eV) }\end{array}$ & $\begin{array}{c}\text { Lines } \\
\text { used }^{\dagger}\end{array}$ & $\begin{array}{c}\text { Observed } \\
\text { ratio }\end{array}$ & $\begin{array}{c}T_{\mathrm{e}} \\
(\mathrm{K})\end{array}$ \\
\hline$[\mathrm{N}$ II $]$ & 14.5 & $5755 / 6584$ & 0.021 & 10700 \\
{$[\mathrm{~S} \mathrm{III}]$} & 23.3 & $6312 / 18.7$ & 0.0 .0475 & 10000 \\
{$[\mathrm{Ar}$ III $]$} & 27.6 & $7136 / 8.99$ & 0.839 & 8800 \\
{$[\mathrm{~N}$ III $]$} & 29.6 & $1750 / 57.3$ & 0.18 & 9400 \\
{$[\mathrm{O}$ III $]$} & 35.1 & $4363 / 5007$ & 0.0056 & 9400 \\
{$[\mathrm{O}$ III $]$} & 35.1 & $1663 / 5007$ & 0.00466 & 9300 \\
{$[\mathrm{O}$ III $]$} & 35.1 & $5007 / 51.8$ & 7.18 & 9700 \\
{$[\mathrm{Ne}$ III $]$} & 41.0 & $3869 / 15.5$ & 0.493 & 9300 \\
\hline
\end{tabular}

$\dagger$ When the wavelength has 4 figures it has the units of Angstrom and with 3 figures it is in microns.

the previous table. It can be seen that the electron temperature is roughly constant as a function of ionization potential. The electron temperature has also been determined from the Balmer jump to be 9300 K. (Liu et al. 2004b). Some PNe show a gradient of electron temperature but if any gradient exists in this nebula, is can only be seen in the slightly higher temperatures for the lowest ionization potentials (presumably formed in the outer regions of the nebula). We shall use a value of $9500 \mathrm{~K}$ as a constant nebular temperature.

\subsection{Ion and element abundances}

The ion abundances have been determined using the following equation:

$\frac{N_{\text {ion }}}{N_{\mathrm{p}}}=\frac{I_{\text {ion }}}{I_{\mathrm{H}_{\beta}}} N_{\mathrm{e}} \frac{\lambda_{\mathrm{ul}}}{\lambda_{\mathrm{H}_{\beta}}} \frac{\alpha_{\mathrm{H}_{\beta}}}{A_{\mathrm{ul}}}\left(\frac{N_{\mathrm{u}}}{N_{\text {ion }}}\right)^{-1}$

where $I_{\mathrm{ion}} / I_{\mathrm{H}_{\beta}}$ is the measured intensity of the ionic line compared to $\mathrm{H} \beta, N_{\mathrm{p}}$ is the density of ionized hydrogen, $\lambda_{\mathrm{ul}}$ is the wavelength of the line, $\lambda_{\mathrm{H}_{\beta}}$ is the wavelength of $\mathrm{H} \beta, \alpha_{\mathrm{H}_{\beta}}$ is the effective recombination coefficient for $\mathrm{H} \beta, A_{\mathrm{ul}}$ is the Einstein spontaneous transition rate for the line, and $N_{\mathrm{u}} / N_{\text {ion }}$ is the ratio of the population of the level from which the line originates to the total population of the ion. This ratio has been determined using a five level atom. The atomic data used is given in the paper of Pottasch \& Beintema (1999). The use of a 5 level atom in the case of iron is justified because collisional rates to higher levels followed by cascade through the lower levels are quite small in comparison to direct collisions to the lower levels.

The results are given in Table 7, where the first column lists the ion concerned, the second column the line used for the abundance determination, and the third column gives the intensity of the line used relative to $\mathrm{H} \beta=100$. The fourth column gives the value of the ionic abundance assuming the ion is formed at $9500 \mathrm{~K}$, while the fifth column gives the ionization correction factor (ICF), which has been determined empirically. Notice that the ICF is unity (or almost unity) for many elements listed in the table: $\mathrm{He}, \mathrm{C}, \mathrm{O}, \mathrm{N}, \mathrm{Ne}, \mathrm{Cl}, \mathrm{Ar}$ and probably Fe. The ICF is large for $\mathrm{Si}, \mathrm{P}, \mathrm{Na}$ and $\mathrm{K}$ for which an important stage of ionization has not been measured. The ICF for Si and P has been determined by comparing the observed ionization stages with those of sulfur which has very similar ionization potential as these two elements. For $\mathrm{Na}$ we note that $\mathrm{Na}^{++}$has a high ionization potential so that higher stages of ionization are quite small. The correction for $\mathrm{Na}^{+}$is however difficult to make and could be overestimated. The correction for $\mathrm{K}$ was made by comparing the observed ionization stages with those of Ar which has very similar ionization potentials. 
Table 7. Ionic concentrations and chemical abundances in NGC 6210.

\begin{tabular}{lccccc}
\hline \hline Ion & $\lambda$ & $\mathrm{I} / \mathrm{H} \beta$ & $N_{\text {ion }} / N_{\mathrm{p}}$ & ICF & $N_{\text {el. }} / N_{\mathrm{p}}$ \\
\hline $\mathrm{He}^{+}$ & 5875 & 13.7 & 0.091 & & \\
$\mathrm{He}^{++}$ & 4686 & 1.5 & 0.0013 & 1.0 & 0.092 \\
$\mathrm{C}^{+}$ & 2324 & 1.54 & $4.4(-6)$ & & \\
$\mathrm{C}^{++}$ & 1909 & 25.2 & $1.03(-4)$ & 1.1 & $1.2(-4)$ \\
$\mathrm{N}^{+}$ & 6584 & 18.7 & $4.3(-6)$ & & \\
$\mathrm{N}^{++}$ & 1750 & 2.7 & $5.58(-5)$ & & \\
$\mathrm{N}^{++}$ & 57.3 & 15.0 & $6.50(-5)$ & 1.0 & $7.0(-5)$ \\
$\mathrm{O}^{+}$ & 3727 & 25.0 & $2.8(-5)$ & & \\
$\mathrm{O}^{++}$ & 5007 & 1060 & $4.64(-4)$ & & \\
$\mathrm{O}^{++}$ & 1663 & 5.05 & $4.1(-4)$ & & \\
$\mathrm{O}^{++}$ & 51.8 & 151 & $4.5(-4)$ & & \\
$\mathrm{O}^{+3}$ & 25.8 & 7.1 & $2.36(-6)$ & 1.0 & $4.9(-4)$ \\
$\mathrm{Ne}^{+}$ & 12.8 & 4.55 & $7.25(-6)$ & & \\
$\mathrm{Ne}^{++}$ & 15.5 & 169 & $1.12(-4)$ & & \\
$\mathrm{Ne}^{++}$ & 3869 & 86.7 & $1.09(-4)$ & 1.0 & $1.2(-4)$ \\
$\mathrm{S}^{+}$ & 6731 & 3.2 & $2.45(-7)$ & & \\
$\mathrm{S}^{++}$ & 18.7 & 25.7 & $2.76(-6)$ & & \\
$\mathrm{S}^{++}$ & 6312 & 1.09 & $3.03(-6)$ & & \\
$\mathrm{S}^{+3}$ & 10.5 & 152 & $4.39(-6)$ & 1.0 & $7.4(-6)$ \\
$\mathrm{Ar}^{+}$ & 6.98 & 0.76 & $9.2(-8)$ & & \\
$\mathrm{Ar}^{++}$ & 8.99 & 14.7 & $1.52(-6)$ & & \\
$\mathrm{Ar}^{++}$ & 21.8 & 0.72 & $1.2(-6)$ & & \\
$\mathrm{Ar}^{++}$ & 7135 & 10.7 & $1.12(-6)$ & & \\
$\mathrm{Ar}^{+3}$ & 4740 & 1.53 & $5.35(-7)$ & & \\
$\mathrm{Ar}^{+3}$ & 7237 & 0.088 & $2.0(-6):$ & 1.0 & $2.3(-6)$ \\
$\mathrm{Cl}^{+}$ & 14.3 & 0.043 & $1.0(-8)$ & & \\
$\mathrm{Cl}^{++}$ & 5538 & 0.469 & $7.59(-8)$ & & \\
$\mathrm{Cl}^{+3}$ & 11.7 & 0.82 & $4.97(-8)$ & & \\
$\mathrm{Cl}^{+3}$ & 20.3 & 0.45 & $3.25(-8)$ & & \\
$\mathrm{Cl}^{+3}$ & 7530 & 0.224 & $5.5(-8)$ & 1.0 & $1.4(-7)$ \\
$\mathrm{Fe}^{++}$ & 22.9 & 2.9 & $9.7(-8)$ & & $:$ \\
$\mathrm{Fe}^{+3}$ & 2836 & 0.83 & $4.3(-7)$ & 1.2 & $6.0(-7)$ \\
$\mathrm{Si}^{+}$ & 34.8 & 0.30 & $1.8(-7)$ & & \\
$\mathrm{Si}^{++}$ & 1888 & 1.71 & $1.37(-6)$ & 2.2 & $3.4(-6)$ \\
$\mathrm{P}^{++}$ & 17.9 & 0.21 & $4.5(-8):$ & $2.2:$ & $1.0(-7):$ \\
$\mathrm{Na}^{++}$ & 7.31 & 0.47 & $3.75(-7)$ & $2:$ & $7.5(-7):$ \\
$\mathrm{K}^{+3}$ & 5.98 & $0.21:$ & $1.14(-8)$ & & \\
$\mathrm{K}^{+3}$ & 6794 & 0.026 & $1.18(-8)$ & $2:$ & $2.4(-8)$ \\
$\mathrm{C}^{++}$ & 4267 & 0.34 & $3.2(-4)$ & & \\
$\mathrm{Mg}^{++}$ & 4481 & 0.020 & $1.9(-5)$ & $1.1:$ & $2.0(-5)$ \\
\hline & & & & & \\
\hline
\end{tabular}

Intensities given with respect to $\mathrm{H} \beta=100$.

: Indicates uncertain value.

Wavelengths in Angstrom for all values of $\lambda$ above 1000, otherwise in $\mu \mathrm{m}$.

The error of measurement of the IRS intensities (see Table 1) is usually small, often not more than $5-7 \%$. In the few cases when the error is large this has either been indicated with a ":" or by not using the line. The correction for adjusting the $\mathrm{SH}$ to LH intensity scales and the diaphragm size is probably small, about $10 \%$. The uncertainty of the collisional strengths introduces an error of $10-15 \%$ so that the total error for the ions of neon, sulfur and argon determined with the IRS measurements is less than $20 \%$. This will also be true of the abundances of these elements because the ICF for these elements is close to unity. The ultraviolet measurements are less certain but only carbon is affected by these measurements. In addition the temperature is more important for the carbon ions and the total errors may be twice as large. The element abundances are given in the last column of Table 7. The recombination line abundances of carbon and magnesium are given at the end of the table. The recombination line abundance of $\mathrm{C}^{++}$is a factor of three higher than the value deduced from the collisionally excited lines at $\lambda 1906 \AA$.
Liu et al. (2004a) have found that this is true for all 12 PNe which they have studied. The reason for this is not yet completely understood. Here as in other recent papers the abundances determined from the collisionally excited lines are used.

The helium abundance was derived using the theoretical work of Benjamin et al. (1999) and Porter et al. (2005). For recombination of singly ionized helium, most weight is given to the $\lambda 5875 \AA$ line, because the theoretical determination of this line is the most reliable.

\section{Comparison with other abundance determinations}

Table 8 shows a comparison of our abundances with the most important determinations in the past years. Several of the elements have never been reported before. Good agreement is found for with the earlier determinations, especially those of Liu et al. (2004a). One of the reasons for this agreement is the fact that no noticeable electron temperature gradient has been found in agreement with the assumption made in the earlier analyses. The $\mathrm{C} / \mathrm{O}$ ratio is clearly less than solar as has been reported earlier. Nitrogen is consistent with being solar in agreement with the value found by Liu et al. (2004a) but somewhat lower than the two other determinations listed. All of these elements as well as $\mathrm{Ne}, \mathrm{Ar}$ and $\mathrm{S}$ are consistent with a solar abundance with a small influence of the galactocentric abundance gradient $(-0.085 \mathrm{dex} / \mathrm{kpc})$ as described for example by Pottasch \& Bernard-Salas (2006). Iron, although the abundance is more uncertain than many of the other elements, is depleted by more than a factor of 30 with respect to the solar abundance, as it is in most of the nebulae already studied. Silicon appears depleted by a factor of $10 . \mathrm{P}, \mathrm{Cl}, \mathrm{K}$ and $\mathrm{Na}$ all seem to be slightly depleted with respect to the Sun by factors between two and three, but the abundances of $\mathrm{P}, \mathrm{K}$ and $\mathrm{Na}$ are more uncertain because only a single ionization stage is observed. The abundance of $\mathrm{Cl}$ as determined in meteorites is a factor of two lower than listed for the Sun. Since the meteorite abundance determination is more reliable than the determination from the solar spectrum $\mathrm{Cl}$ may indeed have the solar abundance. The $\mathrm{Mg}$ abundance given by Liu et al. (2004a) is from a recombination line from the dominant $\mathrm{Mg}^{++}$ion. Mg abundance has been determined in NGC 7662 and NGC 7027 from infrared collisionally excited lines of $\mathrm{Mg}^{+3}$ and $\mathrm{Mg}^{+4}$ which give the same $\mathrm{Mg}$ abundance in these two nebulae as that determined from the recombination line. This $\mathrm{Mg}$ abundance seems therefore to be reliable.

The comparison made in the table with the solar abundance is taken from Asplund et al. (2005). Note that for sulfur and chlorine more weight has been given to the abundance determination in meteorites since this determination is more accurate than for the Sun itself. Neon and argon abundances are taken from the references given in Pottasch \& Bernard-Salas (2006).

\section{Discussion of stellar evolution}

The position of NGC 6210 is about $1 \mathrm{kpc}$ above the galactic plane. This is unusual for a PNe and indicates that it is an old object. This is confirmed by the abundance analysis. The oxygen abundance is similar to the solar value. So is the helium abundance indicating that no helium has been formed in the course of evolution. The nitrogen abundance also has essentially the solar value, indicating that little or no nitrogen has been produced. The low abundance of helium and nitrogen indicate that the second dredge-up may not have taken place. The carbon abundance 
Table 8. Comparison of abundances in NGC 6210.

\begin{tabular}{lrrrrr}
\hline \hline Elem. & Present & $\mathrm{L}^{\dagger}$ & $\mathrm{HKB}^{\dagger}$ & $\mathrm{KH}^{\dagger}$ & Solar $^{\dagger}$ \\
\hline $\mathrm{He}$ & 0.092 & 0.098 & 0.11 & 0.11 & 0.086 \\
$\mathrm{C}(-4)$ & 1.2 & 1.1 & & 0.94 & 2.5 \\
$\mathrm{~N}(-5)$ & 7.0 & 7.4 & 11.4 & 14.5 & 6.1 \\
$\mathrm{O}(-4)$ & 4.9 & 4.4 & 5.2 & 4.4 & 4.6 \\
$\mathrm{Ne}(-4)$ & 1.2 & 1.0 & 1.3 & 0.71 & 1.2 \\
$\mathrm{~S}(-6)$ & 7.4 & 6.5 & 6.8 & & 13.8 \\
$\mathrm{Ar}(-6)$ & 2.3 & 1.3 & 1.8 & & 3.2 \\
$\mathrm{Cl}(-7)$ & 1.4 & 1.25 & 1.4 & & 3.2 \\
$\mathrm{Si}(-5)$ & 0.34 & & & & 3.25 \\
$\mathrm{Fe}(-7)$ & 6.0 & 9.5 & & & 284 \\
$\mathrm{P}(-7)$ & 1.0 & & & & 2.3 \\
$\mathrm{~K}(-8)$ & 2.4 & & & & 12 \\
$\mathrm{Na}(-7)$ & 7.5 & & & & 14.7 \\
$\mathrm{Mg}(-5)$ & & 2.0 & & & 3.4 \\
\hline
\end{tabular}

$\dagger$ References: L: Liu et al. (2004a); HKB: Henry et al. (2004); K: Kwitter \& Henry (1998); Solar: Asplund et al. (2005); except Ne and $\mathrm{Ar}$ (see Pottasch \& Bernard-Salas 2006).

is low, lower than solar although it is more uncertain than some of the other abundances. This low carbon abundance indicates that the third dredge-up has also not taken place and the PN has evolved from a star of low mass. In the evolution models of Karakas (2007) for a solar composition $(Z=0.008)$ the carbon abundance begins to increase somewhere between the $1.5 M_{\odot}$ and $1.75 M_{\odot}$ initial mass models indicating that the initial mass of the star was less than $1.7 M_{\odot}$. The nitrogen abundance, according to these models, begins to increase for the lowest initial mass model, the $1 M_{\odot}$ model, by a factor of 2 , due to the first dredge-up. Since this is more than the observed increase, this indicates that the PN has evolved from a star of initial mass less than $1 M_{\odot}$. If the $Z=0.02$ models were used the predicted nitrogen abundance is smaller and agrees with the observed increase. Because of the uncertainties both in the models and the observations, the best that can be concluded is that the initial mass is $\leq 1 M_{\odot}$.

The abundances of oxygen, sulfur and neon fit well on the abundances as a function of position in the galaxy as given by Pottasch \& Bernard-Salas (2006). The abundances in NGC 6210 are very similar to several elliptical PNe close to the Sun. In particular the abundances are almost the same as in NGC 7662 (Pottasch et al. 2001) and quite similar to NGC 6826 (Surendiranath \& Pottasch 2008). The carbon abundance is lower in NGC 6210 than in other elliptical nebulae studied (see Pottasch \& Bernard-Salas 2006, for a summary). This is certainly due to the low initial mass of the exciting star.

\subsection{Dust}

From the abundances in Table 8 it may be seen that there are a number of elements whose abundance in NGC 6210 is more than a factor of 2 lower than the solar abundance. Since dust is known to be present in the nebula, a probable reason for the low abundance of these elements is that they are in the form of dust. Si, $\mathrm{Fe}$ and $\mathrm{K}$ have especially low abundances, a factor of almost 10 or more. There are other elements with an abundance lower by a factor of about 2. These are $\mathrm{S}, \mathrm{Cl}, \mathrm{P}, \mathrm{Na}$ and $\mathrm{Mg}$. For these latter elements several possibilities could explain the difference. For example the determination of the abundance, either nebular or solar, could be wrong. In fact, because the sulfur abundance in all PNe and HII regions is always lower than the solar value, it has often been argued that the solar value is incorrect (e.g. Pottasch \& Bernard-Salas 2006). If the difference between the measured abundance in NGC 6210 and the expected abundance in $\mathrm{Si}, \mathrm{Fe}$ and $\mathrm{K}$ is in the form of dust, the ratio of dust mass to total mass is $2.6 \times 10^{-3}$. Si and $\mathrm{Fe}$, being refractory, are expected to be associated with dust grains. But because at least some of these elements will be in the form of compounds with other elements this number will certainly be higher. There is no clear indication that a great deal of $\mathrm{Mg}$ is in the form of dust although a small amount of $\mathrm{MgS}$ has been seen in the infrared spectrum of some nebulae. In addition sulfur would then be underabundant. Furthermore there is no indication that oxygen is underabundant and thus part of a dust compound. But if a limited amount of oxygen is tied up in molecules this might not be noticed.

\section{The central star}

\subsection{Stellar temperature}

The central star has been discussed in the introduction and has been studied by several authors. Using the magnitude of the star given there it was concluded that the hydrogen Zanstra temperature is $T_{\mathrm{z}}(\mathrm{H})=51000 \mathrm{~K}$ and the ionized helium Zanstra temperature as $61000 \mathrm{~K}$. The energy balance temperature requires the knowledge of the ratio of the forbidden-line intensities to $\mathrm{H} \beta$ which is essentially the energy above the Lyman limit per ionizing photon. This value is found by summing the intensities given above, and is about 23.2 with only a minimal correction for unmeasured lines. Using Fig. 4 of Preite-Martinez \& Pottasch (1983) and assuming that the star radiates as a blackbody this translates into a temperature of about $69000 \mathrm{~K}$. If a model atmosphere had been used instead of a blackbody, the energy balance temperature would be slightly lower. The energy balance temperature for NGC 6210 has also been given by Preite-Martinez et al. (1991) who find a somewhat lower value of $65700 \mathrm{~K}$. This is because they did not have the infrared measurements and were forced to estimated them. They therefore found a ratio of forbidden line intensity to $\mathrm{H} \beta$ of 21.3 . The low value of the hydrogen Zanstra temperature $\left(T_{\mathrm{z}}(\mathrm{H})\right)$ may be due to the nebula being optically thin to radiation ionizing hydrogen, but it could also be due to departure from blackbody ionizing radiation. An average stellar temperature of about $65000 \mathrm{~K}$ is a reasonable first approximation. This is similar to the conclusion of Tinkler \& Lamers (2002) who estimate a central star temperature of $74000 \mathrm{~K}$.

Using a stellar temperature of $65000 \mathrm{~K}$ and the magnitude and extinction given above, the radius of the star is $R=0.35 \times$ $R_{\odot}$. The luminosity, using the distance $(d=1.57 \mathrm{kpc})$ given in the introduction, is $L=2000 L_{\odot}$.

The question arises as to whether this luminosity is consistent with the initial mass derived from the abundance of elements in Sect. 7. Schonberner (1983) has predicted the evolution of the luminosity of two stars, one with a luminosity of $0.8 M_{\odot}$, the other with a luminosity of $1 M_{\odot}$. The observed luminosity of $2000 L_{\odot}$ at a stellar temperature of $65000 \mathrm{~K}$ lies just between these two predicted curves, thus predicting an initial mass of $M_{\odot}=0.9$. This is very good agreement with the value derived in Sect. 7. On the other hand the curves of Schonberner (1983) predict an age for the nebula of about 40000 years. This is much larger than the value of 2000 years derived from the radius of the nebula $\left(1.7 \times 10^{12} \mathrm{~km}\right)$ and the nebular velocity. There is therefore only a partial agreement between theory and observation. 


\section{Conclusions}

The nebular abundances of fourteen elements have been determined. Of the elements which have not been created in stellar evolution (O, Ne, S, Ar, $\mathrm{Cl}$ and possibly $\mathrm{Si}$ ), O, Ne and Ar have abundances very similar to the solar abundance, while $\mathrm{S}$ and $\mathrm{Cl}$ are about a factor of two lower. The helium abundance is approximately solar; this probably indicates that no helium has been formed during the evolution of the central star. Carbon is lower than solar indicating that no third dredge-up has taken place. Nitrogen also has a solar abundance implying that little nitrogen has been formed, probably in the first dredge-up. A comparison of this low nitrogen abundance with the nitrogen abundance expected from the models of Karakas leads to the conclusion that the central star has evolved from an object having a low initial mass, probably $\leq 1 M_{\odot}$. All of the remaining elements measured have abundances lower than solar. But some (P, $\mathrm{Na}$ and $\mathrm{Mg}$ ) are only a factor of two less than solar, while $\mathrm{Si}, \mathrm{Fe}$ and to a lesser extent K, are an order of magnitude less than solar. These latter elements are likely to be in the form of dust.

The electron temperature is roughly constant throughout the nebula, in contrast to some nebulae in which a strong temperature gradient is found. The luminosity found from considering the central star is in agreement with that predicted for a star of $0.9 M_{\odot}$. The observational age of the nebula is however much lower than the theory predicts.

Acknowledgements. This work is based on observations made with the Spitzer Space Telescope, which is operated by the Jet Propulsion Laboratory, California Institute of Technology under NASA contract 1407. Support for this work was provided by NASA through Contract Number 1257184 issued by JPL/Caltech.

\section{References}

Acker, A., Marcout, J., Ochsenbein, F., et al. 1992, Strasbourg-ESO catalogue Asplund, M., Grevesse, N., \& Sauval, A. J. 2005, ASP Conf. Ser. (Bash \& Barnes)

Becker, R. H., White, R. L., \& Edwards, A. L. 1991, ApJS, 75, 1

Benjamin, R. A., Skillman, E. D., \& Smits, D. P. 1999, ApJ, 514, 307

Bernard Salas, J., Pottasch, S. R., Beintema, D. A., \& Wesselius, P. R. 2001, A\&A, 367, 949

Fluks, M. A., Plez, B., de Winter, D., et al. 1994, A\&AS, 105, 311

Gregory, P. C., \& Condon, J. J. 1991, ApJS, 75, 1011

Hajian, A. R., Terzian, Y., \& Bignell, C. 1995, AJ, 109, 2600

Henry, R. B. C., Kwitter, K. B., \& Balick, B. 2004, AJ, 127, 2284

Higdon, S. J. U., Devost, D., Higdon, J. L., et al. 2004, PASP, 116, 975

Hippelein, H., \& Munch, G. 1981, A\&A, 95, 100

Houck, J. R., Appelton, P. N., Armus, L., et al. 2004, ApJS, 154, 18

Hummer, D. G., \& Storey, P. J. 1987, MNRAS, 224, 801

Karakas, A., \& Lattanzio, J. C. 2007, PASA, 24, 103; and Thesis (Karakas) 2003

Kerber, F., Mignani, R. P., Guglielmetti, F., et al. 2003, A\&A, 408, 1029

Kwitter, K. B., \& Henry, R. B. C. 1998, ApJ, 493, 247

Liu, Y., Liu, X.-W., Barlow, M. J., et al. 2004a, MNRAS, 353, 1251

Liu, Y., Liu, X.-W., Luo, S.-G., et al. 2004b, MNRAS, 353, 1231

Mendez, R. H. 1991, IAU Symp., 145, 375

Phillips, J. P., \& Cuesta, L. 1996, AJ, 111, 1227

Porter, R. L., Bauman, R. P., Ferland, G. J., et al. 2005, ApJ, 622, L73

Pottasch, S. R. 1984, Planetary Nebulae (Dordrecht: Reidel Publ. Co.)

Pottasch, S. R., \& Beintema, D. A. 1999, A\&A, 347, 974

Pottasch, S. R., \& Bernard-Salas, J. 2006, A\&A, 457, 189

Pottasch, S. R., Wesselius, P. R., Wu, C. C., et al. 1978, A\&A, 62, 95

Pottasch, S. R., Beintema, D. A., \& Feibelman, W. A. 2000, A\&A, 363, 767

Pottasch, S. R., Beintema, D. A., Bernard Salas, J., \& Feibelman, W. A. 2001, A\&A, 380, 684

Preite-Martinez, A., \& Pottasch, S. R. 1983, A\&A, 126, 31

Preite-Martinez, A., Koppen, J., Acker, A., et al. 1991, A\&AS, 88, 121

Schonberner, D. 1983, ApJ, 272, 708

Surendiranath, R., \& Pottasch, S. R. 2008, A\&A, 483, 519

Tinkler, C. M., \& Lamers, H. J. G. L. M. 2002, A\&A, 384, 987

Werner, M., Roellig, T. L., Low, F. J., et al. 2004, ApJS, 154, 1 